ISSN 2179-6750

\title{
Análise estrutural da informatização da atenção básica no estado da Bahia, Brasil
}

Structural analysis of computerization of primary care in the state of Bahia, Brazil Análisis estructural de la informatización de la atención primaria en el estado de Bahía, Brasil

Mailson Fontes de Carvalho ${ }^{1}$; Ícaro José Ribeiro dos Santos ${ }^{2}$; Eduardo Nagib Boery ${ }^{3}$; Cristina de Andrade Setenta ${ }^{4}$

\section{Resumo}

Trata-se de um estudo transversal, descritivo, com objetivo de analisar a informatização da atenção básica no estado da Bahia. Foram analisados dados de 3.604 unidades básicas de saúde, considerando variáveis como distribuição regional e porte populacional. O estudo evidencia reduzidos percentuais de informatização das unidades, representada por ausência ou baixo número de computadores e periférico, com menores índices de informatização nas regiões mais distantes da capital e maior percentual de computadores em municípios entre 20 e 50 mil habitantes, destacando aspectos como falta de conectividade e não utilização do programa Telessaúde. Pode-se concluir que ainda há muito a avançar na inserção de equipamentos de informática e de conectividade nos serviços de atenção básica à saúde investigados.

Descritores: Atenção Primária à Saúde, Saúde da Família, Informática em Saúde Pública.

\section{Abstract}

This is a cross-sectional, descriptive study in order to examine the computerization of primary care in the state of Bahia. Data were analyzed for 3604 basic health units, considering variables such as regional distribution and population size. The study shows reduced percentage of units computerization, represented by the absence or low number of computers and peripheral, with lower rates of computerization in the areas far away of the capital and a higher percentage of computers in municipalities between 20 and 50 thousand inhabitants, highlighting aspects such as lack connectivity and non-use of the Telehealth program. It can be concluded that there is still much to advance the integration of computer equipment and connectivity in primary care services to health investigated.

Key-words: Primary Care Health, Family Health, Public Health Informatics.

\footnotetext{
${ }^{1}$ Doutorando em Enfermagem e Saúde (Área de Concentração: Saúde Pública) pela Universidade Estadual do Sudoeste da Bahia (UESB). Professor Assistente da Universidade Federal do Piauí (UFPI). Av. José Moreira Sobrinho, s/n, Jequiezinho, Jequié, Bahia, Brasil. CEP: 45.206-190. Email: mailsoncarvalho@ yahoo.edu.br

${ }^{2}$ Doutorando em Enfermagem e Saúde (Área de Concentração: Saúde Pública) pela Universidade Estadual do Sudoeste da Bahia (UESB). Bolsista Capes. Av. José Moreira Sobrinho, s/n, Jequiezinho, Jequié, Bahia, Brasil. CEP: 45.206-190. Email: icaro.ribeiro29@gmail.com

${ }^{3}$ Doutor em Enfermagem pela Universidade Federal de São Paulo (UNIFESP). Professor Titular da Universidade Estadual do Sudoeste da Bahia (UESB). Av. José Moreira Sobrinho, s/n, Jequiezinho, Jequié, Bahia, Brasil. CEP: 45.206-190.Email: eboery@ig.com.br

${ }^{4}$ Doutora em Saúde Pública pela Universidade de São Paulo (USP). Professora Titular da Universidade Estadual Santa Cruz (UESC). Av. José Moreira Sobrinho, s/n, Jequiezinho, Jequié, Bahia, Brasil. CEP: 45.206-190. Email: cristina70@uol.com.br
} 


\section{Resumen}

Se trata de un estudio transversal, descriptivo con el fin de examinar la informatización de la atención primaria en el estado de Bahía. Se analizaron los datos de 3.604 unidades básicas de salud, teniendo en cuenta variables como la distribución regional y tamaño de la población. El estudio muestra reducida porcentaje de unidades de informatización, representado por la ausencia o el bajo número de ordenadores y periféricos, con tasas más bajas de la informatización en las zonas más alejadas de la capital y un mayor porcentaje de ordenadores en los municipios de entre 20 y 50 mil habitantes, destacando aspectos como la falta la conectividad y la no utilización del programa de Telesalud. Se puede concluir que todavía hay mucho que avanzar en la integración de equipos de cómputo y conectividad en los servicios de atención primaria a la salud investigados.

Palabras-claves: Atención primaria de salud, salud de la família, Informática em Salud Pública.

\section{Introdução}

Em tempos de incorporação tecnológica na área da saúde, é inegável a evolução das práticas de atenção e vigilância à saúde, sobretudo quando estas se utilizam de instrumentos tecnológicos para potencializar e aprimorar diagnósticos, tratamentos e condutas profissionais.

Especialmente nos países em desenvolvimento, as tecnologias de informação e comunicação são colaboradores fundamentais na prestação de cuidados na saúde pública ${ }^{1}$. No Brasil, ainda que muitos tenham sido os avanços para informatização das Unidades de Saúde, ainda existem barreiras que dificultam a utilização de ferramentas tecnológicas, em especial, na área de informática ${ }^{2}$.

Como parte do enfrentamento deste desafio, ainda em 2011 fora instituído o Programa de Requalificação de Unidades Básicas de Saúde (RequalificaUBS), que traz à cena o componente "Informatização e Telessaúde Brasil Redes na Atenção Básica", com objetivo de potencializar o processo de informatização das Unidades Básicas de Saúde (UBS) e desenvolver ações de apoio à atenção à saúde e educação permanente das equipes de atenção básica, na perspectiva da melhoria da qualidade do atendimento através da mudança das práticas de atenção e da organização do processo de trabalho ${ }^{3}$.

No âmbito do RequalificaUBS são classificadas como Equipes de Atenção Básica/Saúde da Família com informatização e conectividade aquelas alocadas em UBS com ponto de Telessaúde e que disponham de computador conectado à internet, kit multimídia e webcam e/ou que disponibilize dispositivos móveis para solicitação de teleconsultorias pelos profissionais da equipe 2,3 .

Ainda que tardiamente, a busca por garantir qualidade nos serviços prestados na atenção básica vem sendo posta em destaque atualmente, especialmente após a criação do Programa de Melhoria do Acesso e da Qualidade da Atenção Básica (PMAQ-AB), que objetiva ampliar o acesso 
ISSN 2179-6750

e a qualidade do cuidado na atenção básica através de monitoramento e avaliação de indicadores, do aprimoramento do processo de trabalho e da avaliação da satisfação dos usuários, induzindo a criação de um ciclo contínuo de melhoria dos serviços vinculados a repasses de recursos destinados a implantação de padrões de qualidade nas equipes de atenção básica ${ }^{4}$.

Nessa perspectiva, programas como o REQUALIFICAUBS e o PMAQ-AB fazem parte de uma série de estratégias governamentais instituídas no âmbito da nova Política Nacional de Atenção Básica, na perspectiva de fomentar mudanças importantes e persistentes em diversas dimensões do processo de trabalho das equipes de atenção básica ${ }^{5}$.

Também são ofertadas novas perspectivas ao trabalho profissional, com a implantação do novo Sistema de Informação em Saúde para a Atenção Básica (SISAB), desenvolvido na perspectiva da utilização do prontuário eletrônico do paciente e de atividades registradas eletronicamente através de ferramentas como computadores, tablets e outros equipamentos eletrônicos que permitam o acesso ao SISAB através da internet.

Considerando a relevância da utilização da informática e da conexão com a internet no aprimoramento do processo de trabalho das equipes de atenção básica e sua destacada importância no fomento aos processos de reorganização e melhoria da qualidade dos serviços em questão, buscou-se caracterizar o atual cenário de informatização das UBS no estado da Bahia.

\section{Método}

Trata-se de estudo descritivo e analítico, utilizando dados provenientes do Censo das Unidades Básicas de Saúde, realizado pelo Ministério da Saúde (MS) com objetivo de levantar informações para melhorar os serviços da Atenção Básica. Este levantamento ocorreu associado ao desenvolvimento da avaliação externa no $1^{\circ}$ ciclo do PMAQ-AB, ocorrido em 2012. Segundo o Ministério da Saúde ${ }^{6}$, foram visitados 5.511 municípios brasileiros, realizando através do censo, a avaliação da infraestrutura de 37.690 UBS.

Para satisfazer aos objetivos dessa investigação, utilizou-se o banco de dados disponibilizado pelo MS em acesso aberto, considerando um recorte de análise que contemplou informações de 3.604 unidades básicas de saúde avaliadas no estado da Bahia.

Maior estado da região nordeste do país, a Bahia possui mais de 14 milhões de habitantes distribuídos em 417 municípios ${ }^{7}$, organizados em 28 Regiões que se aglutinam em 09 Macrorregiões de Saúde. Neste território, segundo dados do Departamento de Atenção Básica do Ministério da Saúde, a cobertura das Equipes de Saúde da Família em 2011 chegava a 61,9\%, atualmente em torno dos $72 \%{ }^{8}$. 
ISSN 2179-6750

Para fins de análise, foram consideradas as questões relativas a informatização das UBS (Bloco I.17 do Instrumento de avaliação externa do PMAQ-AB), analisadas através da apresentação de frequências relativas e absolutas. Os microdados foram transportados para o software SPSS versão 20.0 (Statistical Package for Social Science, SSPS Incorporation, Chicago, IL, USA). Nesse aspecto, a fim de evidenciar associação entre as variáveis assumidas procedeu-se a utilização do teste qui-quadrado, com nível de significância $p \leq 0,05$. A comparação entre a quantidade de computadores nas diferentes regiões foi mensurada por meio do teste de Kruskal Whallis dada a não normalidade da amostra evidenciada pelo teste de Kolmogorov Sminorv.

\section{Resultados}

De início, a análise estrutural da informatização das 3.605 UBS, evidenciou que 67,2\% (n=2420) não possuíam computadores. Observada a distribuição respeitando-se a divisão das regiões de saúde, nota-se que a macrorregião de Jequié apresenta a maior proporção de unidades com computadores $46,1 \%$ ( $n=76)$, o que representa $6,4 \%$ de todo o estado, atrás apenas da região da capital Salvador que apresenta $68,6 \%(n=144)$ das equipes com computadores, perfazendo um total de $12,2 \%$ do Estado.

Tabela 1. Distribuição de computadores em UBS de acordo com as regiões de saúde do estado da Bahia, Brasil, 2012.

\begin{tabular}{l|c|c|c|c}
\hline \multirow{2}{*}{} & \multicolumn{4}{|c}{ Computador } \\
\cline { 2 - 5 } & \multicolumn{2}{|c}{ Sim } & \multicolumn{2}{c}{ Não } \\
\cline { 2 - 5 } & $\mathbf{n}$ & \% & n & \% \\
\hline Alagoinhas & 28 & 2,4 & 111 & 4,6 \\
\hline Irecê & 44 & 3,7 & 117 & 4,8 \\
\hline Itaberaba & 39 & 3,3 & 67 & 2,8 \\
\hline Itabuna & 46 & 3,9 & 82 & 3,4 \\
\hline Itapetinga & 18 & 1,5 & 68 & 2,8 \\
\hline Jacobina & 37 & 3,1 & 104 & 4,3 \\
\hline Jequié & 76 & 6,4 & 89 & 3,7 \\
\hline Juazeiro & 32 & 2,7 & 86 & 3,6 \\
\hline Paulo Afonso & 16 & 1,4 & 80 & 3,3 \\
\hline Porto Seguro & 62 & 5,2 & 31 & 1,3 \\
\hline Ribeira do Pombal & 20 & 1,7 & 86 & 3,6 \\
\hline Barreiras & 41 & 3,5 & 52 & 2,1 \\
\hline Salvador & 144 & 12,2 & 66 & 2,7 \\
\hline Santa Maria da Vitória & 27 & 2,3 & 60 & 2,5 \\
\hline Santo Antônio de Jesus & 47 & 4,0 & 119 & 4,9 \\
\hline Seabra & 10 & 0,8 & 68 & 2,8 \\
\hline Senhor do Bonfim & 30 & 2,5 & 61 & 2,5 \\
\hline Serrinha & 29 & 2,5 & 179 & 7,4 \\
\hline Teixeira de Freitas & 55 & 4,6 & 93 & 3,8 \\
\hline Valença & 9 & 0,8 & 97 & 4,0 \\
\hline Vitória da Conquista & 54 & 4,6 & 123 & 5,1 \\
\hline Brumado & 69 & 5,8 & 112 & 4,6 \\
\hline
\end{tabular}


ISSN 2179-6750

\begin{tabular}{l|c|c|c|c}
\hline Camaçari & 58 & 4,9 & 40 & 1,7 \\
\hline Cruz das Almas & 26 & 2,2 & 63 & 2,6 \\
\hline Feira de Santana & 66 & 5,6 & 222 & 9,2 \\
\hline Guanambi & 57 & 4,8 & 85 & 3,5 \\
\hline Ibotirama & 31 & 2,6 & 28 & 1,2 \\
\hline Ilhéus & 12 & 1,0 & 31 & 1,3 \\
\hline
\end{tabular}

Fonte: Microdados do Censo das UBS. Brasil, 2012.

Todavia, foi possível constatar que 36 unidades referiram a presença de computador sem condições de uso. Desta forma, evidenciou-se média de $1,7( \pm 1,7)$ computadores em condições de uso, variando de 0 (zero) a 25 máquinas.

Levando-se em consideração a disponibilidade de computadores de acordo com o porte populacional, foi evidenciada associação estatisticamente significante, sendo que aproximadamente $29 \%$ dos computadores encontravam-se disponíveis em municípios entre 20 e 50 mil habitantes, como apresentado na tabela 2.

Tabela 2. Distribuição de computadores em UBS de acordo com porte populacional dos municípios do estado da Bahia, Brasil, 2012.

\begin{tabular}{c|r|r|r|r}
\hline \multirow{2}{*}{} & \multicolumn{4}{|c}{ Computador } \\
\cline { 2 - 5 } Porte populacional & \multicolumn{2}{|c|}{ Sim } & \multicolumn{2}{c}{ Não } \\
\hline Até 5000 habitantes & \multicolumn{1}{|c}{$\mathbf{n}$} & \multicolumn{1}{c}{ \% } & \multicolumn{1}{c}{$\mathbf{n}$} & \multicolumn{1}{c}{$\%$} \\
\hline 5.001 a 10.000 habitantes & 88 & 7,6 & 16 & 0,7 \\
\hline 10.001 a 20.000 habitantes & 304 & 25,9 & 738 & 30,9 \\
\hline 20.001 a 50.000 habitantes & 340 & 28,9 & 865 & 36,2 \\
\hline 50.001 a 100.000 habitantes & 95 & 8,1 & 356 & 14,9 \\
\hline 100.001 a 500.000 habitantes & 206 & 17,5 & 184 & 7,7 \\
\hline Mais de 500.000 habitantes & 136 & 11,6 & 67 & 2,8 \\
\hline Fonte: Microdados do Censo das UBS. Brasil, 2012. &
\end{tabular}

Observada a disponibilidade de acesso à internet e do serviço de Telessaúde nas UBS estudadas, pode-se constatar que $15,4 \%(n=556)$ possuíam acesso a rede e $1,6 \%(n=56)$ ao referido programa.

Tabela 3: Distribuição da presença de internet e telessaúde de acordo com as regiões de saúde do estado da Bahia, Brasil, 2012

\begin{tabular}{l|c|c|c|c|c|c|c|c}
\hline \multirow{2}{*}{} & \multicolumn{4}{|c|}{ Internet } & \multicolumn{4}{c}{ Telessaúde } \\
\cline { 2 - 11 } & \multicolumn{2}{|c|}{ Sim } & \multicolumn{2}{c|}{ Não } & \multicolumn{3}{c}{ Sim } & \multicolumn{2}{c}{ Não } \\
\cline { 2 - 10 } & $\mathbf{n}$ & \% & n & \% & n & \% & n & \% \\
\hline Alagoinhas & 8 & 1,4 & 131 & 4,3 & 3 & 5,4 & 136 & 3,8 \\
\hline Irecê & 11 & 2,0 & 150 & 4,9 & 1 & 1,8 & 160 & 4,5 \\
\hline Itaberaba & 20 & 3,6 & 86 & 2,8 & 3 & 5,4 & 103 & 2,9 \\
\hline Itabuna & 32 & 5,8 & 96 & 3,2 & 4 & 7,1 & 124 & 3,5 \\
\hline
\end{tabular}


ISSN 2179-6750

\begin{tabular}{l|c|c|c|c|c|c|c|c}
\hline Itapetinga & 8 & 1,4 & 78 & 2,6 & 0 & 0 & 86 & 2,4 \\
\hline Jacobina & 6 & 1,1 & 135 & 4,4 & 2 & 3,6 & 139 & 3,9 \\
\hline Jequié & 36 & 6,5 & 129 & 4,2 & 4 & 7,1 & 161 & 4,5 \\
\hline Juazeiro & 12 & 2,2 & 106 & 3,5 & 0 & 0 & 118 & 3,3 \\
\hline Paulo Afonso & 6 & 1,1 & 90 & 3,0 & 0 & 0 & 96 & 2,7 \\
\hline Porto Seguro & 45 & 8,1 & 48 & 1,6 & 2 & 3,6 & 91 & 2,6 \\
\hline Ribeira do Pombal & 7 & 1,3 & 99 & 3,2 & 0 & 0 & 106 & 3,0 \\
\hline Barreiras & 28 & 5,0 & 65 & 2,1 & 5 & 8,9 & 88 & 2,5 \\
\hline Salvador & 114 & 20,5 & 96 & 3,2 & 8 & 14,3 & 202 & 5,7 \\
\hline Santa Maria da Vitória & 14 & 2,5 & 73 & 2,4 & 2 & 3,6 & 85 & 2,4 \\
\hline Santo Antônio de Jesus & 14 & 2,5 & 152 & 5,0 & 4 & 7,1 & 162 & 4,6 \\
\hline Seabra & 2 & 0,4 & 76 & 2,5 & 1 & 1,8 & 77 & 2,2 \\
\hline Senhor do Bonfim & 11 & 2,0 & 80 & 2,6 & 2 & 3,6 & 89 & 2,5 \\
\hline Serrinha & 9 & 1,6 & 199 & 6,5 & 0 & 0 & 208 & 5,9 \\
\hline Teixeira de Freitas & 23 & 4,1 & 125 & 4,1 & 5 & 8,9 & 143 & 4,0 \\
\hline Valença & 3 & 0,5 & 103 & 3,4 & 0 & 0 & 106 & 3,0 \\
\hline Vitória da Conquista & 33 & 5,9 & 144 & 4,7 & 3 & 5,4 & 174 & 4,9 \\
\hline Brumado & 17 & 3,1 & 164 & 5,4 & 0 & 0 & 181 & 5,1 \\
\hline Camaçari & 45 & 8,1 & 53 & 1,7 & 1 & 1,8 & 97 & 2,7 \\
\hline Cruz das Almas & 5 & 0,9 & 84 & 2,8 & 0 & 0 & 89 & 2,5 \\
\hline Feira de Santana & 26 & 4,7 & 262 & 8,6 & 1 & 1,8 & 287 & 8,1 \\
\hline Guanambi & 12 & 2,2 & 130 & 4,3 & 2 & 3,6 & 140 & 3,9 \\
\hline Ibotirama & 5 & 0,9 & 54 & 1,8 & 1 & 1,8 & 58 & 1,6 \\
\hline Ilhéus & 4 & 0,7 & 39 & 1,3 & 2 & 3,6 & 41 & 1,2 \\
\hline
\end{tabular}

Uma vez que os computadores para acesso ao Telessaúde devem contar com outros aparelhos para a viabilidade do uso, destaca-se que 96,7\% (n=3486) não possuíam câmeras, 91,85\% $(\mathrm{n}=3310)$ caixa de som e 97,7\% (n=3522) microfones, impossibilitando desta forma a operacionalização do serviço de telessaúde.

\section{Discussão}

Em que pese o baixo percentual de UBS que possui computador $(32,8 \%)$, vale ressaltar que a informatização de serviços de saúde é uma realidade iminente desde o final de década de 90 em todo o Brasil. Todavia, vislumbra-se que o cenário contemporâneo ainda é de contrastes e desigualdades na distribuição das tecnologias de comunicação e informação (TIC) no Sistema Único de Saúde (SUS) ${ }^{9}$.

Neste contexto de contrastes e disparidades, a Bahia é um estado brasileiro com extenso território e, como na maioria dos estados, possui diferentes realidades entre suas regiões de saúde, especialmente no âmbito do desenvolvimento socioeconômico e tecnológico. Considerando a distribuição regional adotada no estudo podemos verificar que as regiões mais distantes da capital possuem menor índice de informatização nas unidades, corroborando com a literatura que aponta uma relação direta deste indicador com o desenvolvimento socioeconômico dos municípios. 
ISSN 2179-6750

É fato que a incorporação de TIC na atenção básica vem caminhando a passos lentos, ainda que no âmbito da estratégia saúde da família esta necessidade venha se tornando iminente. Santos e Ferreira ${ }^{10}$ apontam que equipes de saúde e de gestão dos serviços devem atentar-se que a integração dos dados e disponibilidade de informações confiáveis e oportunas são fundamentais para o atendimento e coordenação da atenção. Espera-se que, tais características dos dados sejam alcançadas por meio da informatização dos processos, e que isto melhore a qualidade dos registros e o retorno da informação para a prática de trabalho do profissional de saúde ${ }^{11}$.

Em estudo acerca do processo de trabalho de gerentes de UBS, Fernandes et al. ${ }^{12}$ reforçam que a indisponibilidade de equipamentos tecnológicos em geral nas unidades, comum na maioria das UBS do estudo, é um grande desafio a ser superado no âmbito da organização dos serviços de atenção básica.

A falta de computadores nas UBS inviabiliza não somente o acesso desta a sistemas informatizados protocolados pelo Ministério da Saúde, mas também dificulta o processo gerencial dos serviços, burocratizando ainda mais o processo de trabalho dos profissionais e gerentes, além de provocar custos desnecessários com o preenchimento repetitivo de inúmeros cadastros de usuários 13

É fato que o Ministério da Saúde vem buscando implementar soluções tecnológicas como forma de solucionar alguns entraves para o alcance da qualidade dos serviços. Entre estas, a utilização do Telessaúde e do recém lançado SISAB vem se destacando como mecanismos de qualificação profissional através da educação permanente e de integração e desburocratização dos registros nas UBS.

A meta estipulada pelo Ministério da Saúde foi garantir informatização e conectividade de, no mínimo, 70\% das Equipes de Atenção Básica/Saúde da Família após a conclusão da primeira etapa dos projetos atrelados ao Programa Telessaúde Brasil Redes e Requalifica UBS ${ }^{2}$. Porém, a dificuldade estrutural e de conectividade se impõem ainda como desafios para a efetivação e alcance dos objetivos fundamentais destes projetos, ainda que seja incontestável a sua expansão nos últimos anos ${ }^{13}$.

Para o funcionamento adequado, o Programa Telessaúde Brasil Redes, como atualmente intitula-se, é indica que para a realização das atividades previstas no programa, entre as quais estão a teleconsultoria síncrona e assíncrona, o telediagnóstico e outros processos de educação permanente, são fundamentais além do computador padrão e do acesso à internet, periféricos como câmera e caixas de som. 
Castro Filho et al. ${ }^{14}$ ressaltam a efetivação do processo de telessaúde não se resume apenas a interação virtual entre um solicitante e um teleconsultor por meio da internet, mas uma importante ferramenta para capacitação e aprimoramento profissional. Os autores em questão apontam que a instalação de um núcleo de telessaúde envolve uma estrutura complexa que envolve recursos humanos, equipamentos, aplicativos, estrutura de rede e espaços físicos adequados em todos os pontos de interação, em especial nas UBS.

Nesse processo, podemos destacar que a instalação do Telessaúde na Bahia ocorreu em 2011, com objetivo de implantação de pontos de telessaúde em 296 municípios e um núcleo técnico-científicos único, coordenado pela secretaria Estadual de Saúde da Bahia (SESAB), em um projeto único, com a parceria da SESAB, Fundação Estatal de Saúde da Família (FESF), Universidade Federal da Bahia (UFBA) e secretarias Municipais de Saúde ${ }^{15}$.

Ainda que seja possível constatar que cerca de $70 \%$ dos municípios do estado tenham recebidos recursos fundo a fundo para a implantação de pontos do telessaúde, podemos constatar que ainda em 2012, apenas 56 pontos de telessaúde estavam em funcionamento, com destaque para as regiões de Brumado, Cruz das Almas, Juazeiro, Itapetinga, Paulo Afonso, Serrinha, Ribeira do Pombal e Valença que não apresentaram relato de utilização do Telessaúde, apesar de possuírem UBS com acesso a internet.

Espera-se que a situação atual tenha evoluído, visto que a primeira etapa do Programa Telessaúde Brasil Redes Bahia se propôs a garantir o kit de equipamentos para telessaúde a 740 UBS no estado, e que foram empenhados mais de 3 milhões de reais advindos de recursos do referido programa para a implantação de 05 Núcleos Técnico Científicos e 124 pontos de telessaúde em UBS dos mais diversos municípios do estado ${ }^{16}$.

Faz-se necessário considerar a recente implantação do Núcleo Técnico Científico (NTC) no estado, ocorrido um ano antes da realização do Censo que coletou os dados deste estudo. Porém, há de se ressaltar que há muito desconhecimento acerca das possibilidades de uso e das potencialidades advindas do Programa Telessaúde, ainda que haja relatos de divulgação e apoio institucional para implantação dos pontos em todos os municípios ${ }^{15}$.

Assim, corroborando os achados deste estudo, Silva e Moraes ${ }^{9}$ ao realizarem uma análise acerca da utilização das TIC na população em geral, afirmam que o acesso à internet local, à telefonia, à TV interativa e aos meios eletrônicos fundamentais para realizar ações de telessaúde, ainda são limitados, não tendo ainda a capilaridade em extensão territorial adequada às necessidades do SUS. 


\section{ISSN 2179-6750}

Nota-se que há grande necessidade de informatizar a rede pública e capacitar os profissionais para trabalhar com programas e dados epidemiológicos, a fim de efetivar o novo modelo de atenção básica e de reconhecer situações de vulnerabilidade social e em saúde ${ }^{12}$.

A superação das barreiras físicas e geográficas a partir do uso de tecnologia em práticas de saúde torna essa ferramenta valiosa e indispensável para formação, atualização e ampliação da prática profissional em saúde. Assim, especialmente no estado da Bahia, que possui grande extensão territorial, a utilização de tecnologias de comunicação pode auxiliar a diminuir as desigualdades observadas.

\section{Conclusões}

O estudo evidenciou baixos percentuais de informatização das UBS no estado da Bahia, representados por ausência ou baixo número de computadores e periféricos (i.e. câmeras, caixas de som e microfones), falta de conectividade e não credenciamento ao programa de teleconferências Telessaúde.

Ainda que possamos reafirmar que a tecnologia pode encurtar distâncias e diminuir desigualdades existentes nos serviços, ainda há muito a avançar na inserção de equipamentos de informática e de conectividade, que garantam a possibilidade de incorporação de tecnologias e estratégias de melhoria da atenção à saúde, tais como sistemas de informação on line, prontuários eletrônicos, teleconsultorias, educação permanente, entre outras.

Salienta-se a possibilidade do acompanhamento do processo de informatização dos serviços de atenção básica à saúde através de análises futuras a partir dos microdados dos novos ciclos do PMAQ-AB, para fins de comparação da situação aqui apresentada com dados mais atualizados advindos destas novas avaliações.

\section{Referências}

1. Organização Mundial da Saúde (OMS). kit de ferramentas de estratégia nacional de Telessaúde.2012 [acesso em 02 fev. 2016]. Disponível em: <http://www.itu.int/dms_pub/itud/opb/str/D-STR-E_HEALTH.05-2012-PDF-E.pdf> .

2. Contreras Pinochet L, Lopes A, Silva J. Inovações e Tendências Aplicadas nas Tecnologias de Informação e Comunicação na Gestão da Saúde. Revista de Gestão em Sistemas de Saúde. 2014; 3(2), 11-29. Disponível em: http://www.revistargss.org.br/ojs/index.php/rgss/ article/view/88

3. Ministério da Saúde (Brasil). Universidade Federal do Rio Grande do Sul. Manual de 186 
ISSN 2179-6750

Telessaúde para Atenção Básica / Atenção Primária à Saúde. Brasília: Ministério da Saúde; 2012.

4. Ministério da Saúde (Brasil). Programa de Melhoria da qualidade da Atenção Básica: manual instrutivo. Brasília: Ministério da Saúde; 2011.

5. Pinto HA. Informe Técnico Institucional. O programa nacional de melhoria do acesso e da qualidade e o processo de trabalho das equipes de Atenção Básica. Tempus Actas de saúde coletiva. 2012; 6(2): 315-318.

6. Ministério da Saúde (Brasil). Histórico de cobertura de saúde da família. 2015 [acesso em 30 dez 2015]. Disponível em http://dab.saude.gov.br/portaldab/historico_cobertura_sf.php.

7. Instituto Brasileiro de Geografia e Estatística. Censo demográfico 2010. Rio de Janeiro: IBGE; 2010.

8. Ministério da Saúde (Brasil). Programa de Melhoria do Acesso e Qualidade da Atenção Básica. 2015 [acesso em $30 \mathrm{dez}$ 2015]. Disponível em http://dab.saude.gov.br/ portaldab/cidadao_pmaq2.php.

9. Silva AB, Moraes IHS. O caso da Rede Universitária de Telemedicina: análise da entrada da telessaúde na agenda política brasileira. Physis. 2012; 22 (3):1211-35.

10. Santos DC, Ferreira JBB. O prontuário da família na perspectiva da coordenação da atenção à saúde. Physis. 2012; 22 (3):1121-1137.

11. Gava M, Ferreira LS, Palhares D, Mota ELA. Incorporação da tecnologia da informação na Atenção Básica do SUS no Nordeste do Brasil: expectativas e experiências. Ciênc. saúde colet. 2016. 21(3):891-902.

12. Fernandes LCL, Machado RZ, Anschau GO. Gerência de serviços de saúde: competências desenvolvidas e dificuldades encontradas na atenção básica. Ciênc. saúde colet. 2009; 14 (supl.1): 1541-52.

13. Oliveira DG, Frias PG, Vanderlei LCM, Vidal SA, Novaes MA, Souza WV. Análise da implantação do Programa Telessaúde Brasil em Pernambuco, Brasil: estudo de casos. Cad Saúde Pública. 2015; 31(11):2379-89.

14. Castro Filho ED, Harzheim E, Schmitz CAA, Siqueira ACS. Telessaúde para Atenção Primária. In: Gusso G, Lopes JMC, organizadores. Tratado de medicina de família e comunidade: princípios, formação e prática. Porto Alegre: Artmed; 2012. p.395-403.

15. Piropo TGN, Amaral HOS. Telessaúde, contextos e implicações no cenário baiano. Saúde em debate. 2015; 39 (104):279-287.

16. Secretaria Estadual de Saúde (Bahia). Resolução CIB no 32, de 25 de fevereiro de 2013. 
ISSN 2179-6750

2013[acesso em 05 abr 2016]. Disponível em: http://www.telessaude.ba.gov.br/telessaudeba /legislacao/ 